1. Rodríguez Artalejo F, Banegas Banegas JR, Guallar-Castillón P. Epidemiología de la insuficiencia cardiaca. Rev Esp Cardiol 2004; 57: 163 170.

2. Brophy JM, Joseph L, Rouleau JL. Beta-blockers in congestive heart failure. A Bayesian metaanalisis. Ann Intern Med 2001; 134: 50-60.

3. Sinn DD, McAlister FA. The effects of beta-blockers on morbidity and mortality in a population based cohort of 11.942 elderly patients with heart failure. Am J Med 2002; 113: 650-656.

4. Román-Sánchez P, Conthe P, García-Alegría J, Fortaleza-Rey J, Montero M, Montoto C, for the Herat Failure Working Group pf The Spanish Society of Internal Medicine. Factors influencing medical treatment of heart failure patients in Spanish internal medicine departments: a national survey. Q J Med 2005; 98: 127-138.

5. Witham MD, Gillespie ND, Struthers AS. Age is not a significant risk factor for failed trial of betablocker therapy in older patients with chronic heart failure. Age and Ageing 2004; 33: 467-472.

6. Flatcher MD, Shibata MC, Cotas AJS, Van Veldhuisen JV, Parkhomenko A, Borbola J, et al. Randomized trial to determine the effect of nevibolol on mortality and cardiovascular hospital admission in elderly patients with heart failure (SENIORS). Eur Heart J 2005; 26: 215-222.

7. Urrutía A, Lupón J, Altimir S, González B, Herreros J, Díez C, et al. Uso de bloqueadores beta en pacientes ancianos con insuficiencia cardíaca. Med Clin (Barc) 2006; 126: 206-210.

8. Anguita Sánchez M y los investigadores del registro BADAPIC. Características clínicas, tratamiento y morbimortalidad a corto plazo de pacientes con insuficiencia cardíaca controlados en consultas específicas de insuficiencia cardíaca. Resultados del registro BADAPIC. Rev Esp Cardiol 2004; 57: 1159-1169.

\section{Streptococo agalactiae y taponamiento pericárdico}

\section{Sr. Director:}

El Estreptococo agalactiae (EA), estreptococo del grupo B de Lancefield, forma parte de la flora habitual del tracto gastrointestinal desde donde coloniza al aparato genitourinario. Las infecciones por EA son frecuentes durante la gestación y el puerperio. En este periodo puede producir infecciones tanto en la mujer (infección de la vía urinaria, endometritis, corioamnionitis, y bacteriemia periparto) como en el feto (sepsis, neumonía, meningitis, etc.). Las manifestaciones en adultos fuera de estas dos situaciones son menos frecuentes. Recientemente hemos tenido oportunidad de atender a una paciente con bacteriemia por EA que cursó con afectación multiorgánica incluyendo taponamiento pericárdico.

Mujer de 64 años con antecedentes de hipertensión arterial, diabetes mellitus tipo 2 diagnosticada dos meses antes y en tratamiento dietético, había tenido lesión ulcerosa en pierna izquierda resuelta una semana antes del inicio de su actual sintomatología. Acudió a otro hospital por fiebre de 4 días de evolución que se acompañó de astenia, malestar general y dolor abdominal, todo ello agravado el día del ingreso. Exploración física: tensión arterial 120/70 mmHg. Auscultación cardiopulmonar: taquicardia sinusal. El abdomen era blando con dolor difuso. Analíticamente: glucosa $422 \mathrm{mg} / \mathrm{dl}$, función renal e ionograma normales, proteína $\mathrm{C}$ reactiva $23 \mathrm{mg} / \mathrm{dl}$, bilirrubina total $2,46 \mathrm{mg} / \mathrm{dl}$, GOT $37 \mathrm{U} / \mathrm{l}$, GPT 72 U/l, amilasa 17 U/l. Hemograma: hemoglobina 11,4 g/dl, leucocitos 10.800/ul (87\% PMN), plaquetas 125.000/ul. Estudio de coagulación normal. Radiografía de torax normal. Ecografía abdominal: hígado de estasis. Se realizaron hemocultivos y se inició tratamiento endovenoso con amoxicilina/clavulánico. Éstos fueron positivos a las 48 horas para EA sensible al antibiótico pautado. Setenta y dos horas tras el ingreso la enferma pre- sentó signos de insuficiencia cardiaca con inestabilidad hemodinámica. Se realizó TAC toraco-abdominal y un ecocardiograma que demostraron la presencia de derrame pericárdico y pleural en tercio inferior de ambos hemitorax, por lo que se remitió a nuestro centro.

Al ingreso debido a la presencia de taponamiento cardiaco precisó atención en la Unidad de Medicina Intensiva (UMI), realizándose pericardiocentesis de forma urgente; se obtuvo un líquido hemático con 180.000 hematies, 35.500 leucocitos $(95 \%$ PMN), glucosa $128 \mathrm{mg} / \mathrm{dl}$, proteínas 5,2 g/dl, LDH $2.933 \mathrm{u} / \mathrm{l}$, ADA 3 U/l. Analítica en UMI: se objetiva hiperglucemia leve, función renal y hepática normales e hipoalbuminemia. Hemograma: anemia leve normocítica y leucocitosis con neutrofilia. Estudio de coagulación normal. El análisis del líquido pleural fue compatible con un trasudado. Tras su estabilización clínica la paciente fue trasladada a Medicina Interna, donde de se realizaron los siguientes estudios: serología a virus hepatitis $\mathrm{B}$ y $\mathrm{C}$, VIH, toxoplasma, micoplasma, coxiella, legionella y chlamydia negativas; estudio de autoinmunidad negativo, hormonas tiroideas normales. Ecocardiograma y TAC torácico con mejoría radiológica progresiva hasta la desaparición del líquido en las cavidades pleural y pericárdica. Enema opaco y gastroscopia sin objetivar patología. Examen ginecológico normal. La paciente evolucionó favorablemente siendo dada de alta, se revisó a los tres meses encontrándose asintomática.

Clásicamente la infección por EA se limitaba al periodo de gestación y puerperio. Cada vez es más frecuente la aparición infecciones por este microorganismo afectando diferentes órganos y poblaciones más allá de las señaladas. Los adultos afectados suelen tener enfermedades predisponentes (diabetes mellitus, cirrosis hepática, insuficiencia cardiaca, insuficiencia renal, cáncer de mama, alteraciones neurológicas, úlceras de decúbito entre otras). Las manifestaciones clínicas más frecuentes son las infecciones de piel y tejidos blandos junto con bacteriemias sin foco evidente. Igualmente se ha descrito de forma más infrecuente: endocarditis, infecciones del tracto urinario, meningitis, infecciones osteoarticulares, neumonía y peritonitis.

Nuestra paciente inicialmente presentó un cuadro febril con bacteriemia que evolucionó a pesar de tratamiento antibiótico, a insuficiencia cardiaca por taponamiento pericárdico que precisó pericardiocentesis. Dado el número de leucocitos y el porcentaje de neutrófilos $(95 \%)$ creemos que la afección pericárdica se debió a la acción directa del EA. El derrame pleural, dado que se trataba de un trasudado, se puede explicar por el fallo cardiaco debido al taponamiento pericárdico. Se realizó una investigación exhaustiva para descartar otras etiologías de pericarditis. En la revisión bibliográfica hemos encontrado dos casos en que la afectación por EA cursó con derrame y taponamiento pericárdico, en ambos casos eran diabéticos y tenían un antecedente de lesión dérmica. Estas situaciones se dieron de igual forma en nuestra paciente.

\section{J. D. Mosquera Lozano, A. D. Tejada Evans, A. Echeverría Echeverría, A. Moreno Ortigosa}

Servicio de Medicina Interna. Unidad de Medicina Intensiva. Complejo Hospitalario San Millán-San Pedro. Logroño. La Rioja

1. Wessels MR, Kasper DL. The Changing Spectrum of Group B Streptococcal Disease. N. Engl J Med 1993; 328: 1843-1844.

2. Colford JM, Mohle-Bohetani J, Voste KI. Group B streptococal bacteriemia en adults. Medicine 1995; 74: 176-190.

3. Asad Karim M, Bach RG, Dressler F, Caracciolo E, Donohue TJ, Kern MJ. Purulent pericarditis caused by group B Streptococcus with pericardial tamponade Am Heart J. St Louis, Missouri 1993; 126: 727-730.

4. Álvarez Navascués R, Sánchez Cembellín M, Carrio Montes LI, Quiñones Ortiz L, Guerediaga Madariaga J. Pericarditis purulenta por Streptococcus agalactiae. An Med Interna (Madrid) 2005; 22: 198. 